\title{
Associação do estado nutricional com a hipertensão arterial de adultos
}

\author{
Anderson Zampier Ulbrich ${ }^{1}$ \\ Renata Labronici Bertin ${ }^{2}$ \\ Antonio Stabelini Neto ${ }^{3,4}$ : \\ Rodrigo Bozza ${ }^{4}$ \\ Thiago Silva Piola ${ }^{4}$ \\ Wagner de Campos ${ }^{4}$ \\ ${ }^{1}$ CEFID da Universidade do Estado de Santa Catarina, Florianópolis, SC, Brasil e \\ Bolsista CAPES. \\ ${ }^{2}$ Universidade Regional de Blumenau - FURB, Blumenau, SC, Brasil \\ ${ }^{3}$ Universidade Estadual do Norte do Paraná, Jacarezinho, PR, Brasil \\ ${ }^{4}$ Universidade Federal do Paraná, Curitiba, PR, Brasil
}

\begin{abstract}
Resumo: Objetivo: associar a hipertensão arterial por meio do sexo, estado nutricional e avanço da idade em adultos. Métodos: estudo transversal com 5.053 adultos de ambos os sexos (20 a 60 anos), da cidade de Curitiba, PR. O índice de massa corporal e pressão arterial foram classificados segundo recomendações da Organização Mundial da Saúde e Sociedade Brasileira de Hipertensão. Utilizou-se método descritivo, distribuição de freqüência, ANOVA one way e regressão logística binária para obtenção de "Odds Ratio", com $p<0,05$. Resultados: verificou-se elevada prevalência de indivíduos com sobrepeso $(41,6 \%)$ e obesidade (31,2\%). Entre os obesos, $15,7 \%$ dos homens e $13,8 \%$ das mulheres foram considerados hipertensos e apresentaram quatro vezes mais razão de chances de ter hipertensão quando comparados a seus pares com peso esperado. Conclusão: os homens com o avanço da idade e excesso de peso apresentam associação linear com o aumento da pressão arterial.
\end{abstract}

Palavras-Chave: Índice de Massa Corporal. Hipertensão arterial. Adultos.

\section{Association of the nutritional status with hypertension in adults}

Abstract: Objectives: associate hypertension although gender, nutritional state and advance of age in adults. Materials and methods: A cross-sectional study of 5053 adults of both gender (20 and 60 years) in the city of Curitiba in Brazil. Body mass index and blood pressure were classified according recommendations of the World Health Organization and the Brazilian Society of Hypertension. Used descriptive method, frequency distribution, one-way ANOVA and binary logistic regression to obtain the "Odds Ratio", with $p<0,05$. Results: was a high occurrence of overweight (41.6\%) and obesity (31.2\%). Among obese, $15,7 \%$ is men and $13,8 \%$ is women had hypertension, in which were four times more to has hypertension when compared with normal subjects. Discussion: mans with age advance and overweight presentation association with increased blood pressure.

Keywords: Body mass index. Hypertension. Adults.

\section{Introdução}

Atualmente a hipertensão arterial sistêmica (HAS) tem sido considerada problema de saúde pública de etiologia multifatorial, descrita como um dos maiores fatores de risco prevalentes para o desenvolvimento de doenças cardiovasculares, cerebrovasculares e insuficiência cardíaca congestiva, entre outras doenças do aparelho circulatório em indivíduos adultos (SCHOCKEN et al., 2008).

Estudos observaram que com o aumento do peso corporal, têm-se verificado o aumento e rigidez da parede intima arterial (SCUTERI et al.,
2004; FERREIRA et al., 2005; SCHILLACI et al., 2005; SAFAR; CZERNICHOW e BLACHER, 2006) e mudanças hemodinâmicas tais como débito cardíaco e fração de ejeção do ventrículo esquerdo (STELFOX et al., 2006).

A HAS, recentemente, é responsável em média por $30 \%$ das mortes em indivíduos adultos no mundo (SCHOCKEN et al., 2008) e no Brasil as taxas de prevalência na população brasileira adulta, variam entre $22,3 \%$ a $43,9 \%$ (SOCIEDADE BRASILEIRA DE CARDIOLOGIA (SBC), 2006). A Sociedade brasileira de cardiologia (2006) ainda destacou pesquisas apontando a grande 
ascendência de hipertensão arterial entre a população que está acima do peso, tanto para os homens como para as mulheres. Mais recentemente Chirinos et al. (2009) demonstraram, através dos dados do National Health and Nutrition Examination Survey (NHANES) de 1999-2004, que o Índice de Massa Corporal (IMC) em estudos populacionais é um significante preditor para o aumento da pressão arterial, tendo uma razão de chances (odds ratio=OR) de $\mathrm{OR}=1,04$, para cada cinco unidades do IMC.

Neste contexto, a prevenção e os tratamentos da hipertensão advêm de mudanças no estilo de vida, maior envolvimento com a prática regular de exercícios físicos, alimentação adequada, diminuição dos níveis de estresse, controle do estado nutricional, entre outros fatores modificáveis que contribuem na redução significativa da mortalidade e morbidade cardiovascular da população adulta (POLANCZIK, 2002; GREENLUND; CROFT; MENSAH; 2004).

Diante disso, este estudo objetivou verificar a associação da hipertensão arterial com estado nutricional em diferentes faixas etárias de adultos para ambos os sexos.

\section{Materiais e Métodos}

\section{População}

O estudo de caráter transversal realizado com 5.053 adultos, sendo 2.954 mulheres e 2.099 homens, entre vinte e 60 anos de idade, residentes na zona urbana da cidade de Curitiba, Paraná, Brasil no ano de 2006. Os dados foram obtidos a partir do banco de dados do projeto "Caminha Paraná" desenvolvido pela Secretaria de Esporte e Lazer do estado do Paraná. As idades foram subdivididas por faixa etárias sendo: 20 a 29,99 anos; 30 a 39,99 anos; 40 a 49,99 anos; 50 a 60 anos.

O presente estudo foi conduzido de acordo com a resolução 196/96 do Conselho Nacional de Saúde, seguindo normas do Comitê de Ética de Pesquisa em seres humanos do Setor de Ciências da Saúde da Universidade Federal do Paraná.

\section{Instrumentos e Procedimentos}

As medidas antropométricas (massa corporal e estatura) foram coletadas por professores de Educação Física, treinados. A medida da massa corporal foi realizada em uma balança digital, portátil, com capacidade máxima de $150 \mathrm{~kg}$ e escala de 100g (Balança Pessoal Filizola PL 180), com o indivíduo descalço e usando roupas leves. A estatura foi mensurada através do estadiômetro portátil (Estadiômetro Compacto marca Wiso), graduado em milímetros. As padronizações e aferições dessas medidas antropométricas seguiram as recomendações de Lohman, Roche e Martorell (1988).

Através da divisão da massa corporal pela estatura em metros ao quadrado, foi calculado o Índice de Massa Corporal (IMC). Os valores do IMC foram categorizados, segundo a proposta da World health organization (2000), seguindo os pontos de corte: baixo peso: abaixo de 18,5 $\mathrm{Kg} / \mathrm{m}^{2}$; normal: entre 18,5 a $24,99 \mathrm{Kg} / \mathrm{m}^{2}$; sobrepeso: 25 a 29,99 $\mathrm{Kg} / \mathrm{m}^{2}$; obesidade: acima de $30 \mathrm{Kg} / \mathrm{m}^{2}$.

A pressão arterial (PA) foi aferida conforme protocolo estabelecido nas " $\mathrm{V}$ Diretrizes Brasileiras de Hipertensão" (SOCIEDADE BRASILEIRA DE CARDIOLOGIA, 2006), mediante a utilização de esfigmomanômetro aneróide - e estetoscópio. Todos os indivíduos deveriam permanecer na posição sentada por pelo menos cinco minutos, as pernas descruzadas, pés apoiados no chão e dorso recostado na cadeira. O braço estava posicionado ao nível do coração, com a palma da mão voltada para cima e o cotovelo devidamente fletido. $O$ manguito do esfigmomanômetro foi posicionado ao redor do braço, de 2 a 3 centímetros da fossa cubital, alinhando a campânula do estetoscópio debaixo do espaço antecubital sobre a artéria braquial. Foram realizadas duas aferições com intervalo mínimo de três minutos entre as mesmas, e os valores médios foram utilizados para análises.

Para a realização das avaliações da PA os indivíduos foram orientados a não se exercitar fisicamente 60 a 90 minutos antes da avaliação; não ter ingerido bebidas com cafeína, alcoólicas e não ter fumado nos 30 minutos antecedentes a realização da mesma. Foi considerada pressão arterial sistólica (PAS) no aparecimento do primeiro ruído (fase I de korotkoff) e pressão arterial diastólica (PAD) com o desaparecimento do ruído (SOCIEDADE BRASILEIRA DE CARDIOLOGIA, 2006).

Os pontos de corte da PAS e PAD foram préestipulados como: normal (PAS $<130 \mathrm{mmHg}$ e PAD < $85 \mathrm{mmHg}$ ); limítrofe (PAS $\geq 130$ a $<140$ $\mathrm{mmHg}$ e $P A D \geq 85$ a $\leq 89 \mathrm{mmHg}$ ); hipertensão 
(PAS $\geq 140 \mathrm{mmHg}$ e $P A D \geq 90 \mathrm{mmHg}$ ) (SOCIEDADE BRASILEIRA DE CARDIOLOGIA, 2006).

\section{Análise Estatística}

A estatística descritiva foi empregada no presente estudo para caracterização da amostra, subdividida em faixas etárias, sendo seus valores expressos em médias e desvios padrão. As diferenças entre as faixas etárias foram observadas por meio da análise de variância (ANOVA one way) com post hoc Bonferroni.

A distribuição de freqüência foi utilizada para obtenção da prevalência do estado nutricional e para a obtenção da prevalência de hipertensão arterial conforme as faixas etárias com estado nutricional.
A estimativa da razão de chances (OR) tanto para hipertensão arterial sistólica como para a diastólica foi realizada por meio das variáveis explicativas: sexo, idade categorizada, e estado nutricional. Esta foi realizada pela análise de regressão logística binária pelo método de Backward de Wald, utilizando como pontos de referência para análise: sexo feminino, a idade de 20 a 29,99 anos e IMC entre $18,5 \mathrm{~kg} / \mathrm{m}^{2}$ a 24,99 $\mathrm{kg} / \mathrm{m}^{2}$. O intervalo de confiança adotado foi de $95 \%$ e nível de significância de $p<0,05$. Os procedimentos foram analisados através do programa Statistical Package for the Social Sciences (SPSS) for Windows versão 17.0.

\section{Resultados}

A caracterização da amostra para os homens (Tabela 1) e para as mulheres (Tabela 2) foram expressas em valores médios e desvio padrão segundo a subdivisão por faixa etária, sendo verificadas diferenças entre as mesmas por meio da análise de variância.

Os resultados gerais percentuais do estado nutricional apontam que $42,7 \%$ dos homens e $55 \%$ das mulheres da amostra estão dentro da faixa de normalidade. O sobrepeso foi observado em $41,6 \%$ e $31,2 \%$ para homens e mulheres, respectivamente, assim como a presença de indivíduos obesos foi verificada em $15,7 \%$ dos homens e 13,8\% das mulheres. Já a distribuição pelos pontos de corte do IMC, de acordo com faixas etárias em ambos os sexos está disposta na figura 1.

Tabela 1. Caracterização amostral dos homens, com valores de média e desvio padrão, conforme as categorias de idade.

\begin{tabular}{ccccccc}
\hline \multicolumn{7}{c}{ Idade } \\
\cline { 2 - 7 } & $\begin{array}{c}\mathbf{2 0 - 2 9} \\
\mathbf{( N = 6 2 2})\end{array}$ & $\begin{array}{c}\mathbf{3 0 - 3 9} \\
\mathbf{( N = 6 3 8 )}\end{array}$ & $\begin{array}{c}\mathbf{4 0 - 4 9} \\
(\mathbf{N}=\mathbf{6 8 1})\end{array}$ & $\begin{array}{c}\mathbf{5 0 - 5 9} \\
\mathbf{( N = 4 6 8 )}\end{array}$ & $\mathbf{F}$ & $\mathbf{P}$ \\
\hline Estatura & $171,6 \pm 9,0$ & $170,0 \pm 9,2^{\mathrm{a}}$ & $168,9 \pm 9,2^{\mathrm{a}}$ & $166,2 \pm 9,8^{\mathrm{a}, \mathrm{b}, \mathrm{c}}$ & 30,51 & 0,000 \\
MC & $71,3 \pm 12,7$ & $75,1 \pm 14,2^{\mathrm{a}}$ & $77,6 \pm 14,2^{\mathrm{a}, \mathrm{b}}$ & $76,3 \pm 13,2^{\mathrm{a}}$ & 24,55 & 0,000 \\
IMC & $24,1 \pm 3,6$ & $25,8 \pm 3,8^{\mathrm{a}}$ & $27,1 \pm 4,1^{\mathrm{a}, \mathrm{b}}$ & $27,55 \pm 3,9^{\mathrm{a}, \mathrm{b}, \mathrm{c}}$ & 88,10 & 0,000 \\
PAD & $74,9 \pm 9,0$ & $76,5 \pm 9,1$ & $79,4 \pm 10,2^{\mathrm{a}, \mathrm{b}}$ & $82,69 \pm 11,4^{\mathrm{a}, \mathrm{b}, \mathrm{c}}$ & 49,65 & 0,000 \\
PAS & $116,3 \pm 11,1$ & $117,3 \pm 11,5$ & $121,0 \pm 13,2^{\mathrm{a}, \mathrm{b}}$ & $128,36 \pm 17,5^{\mathrm{a}, \mathrm{b}, \mathrm{c}}$ & 75,11 & 0,000 \\
\hline
\end{tabular}

$\mathrm{N}$ : número de indivíduos; Estatura (cm) MC: Massa Corporal (kg); IMC: índice de massa corporal $\left(\mathrm{kg} / \mathrm{m}^{2}\right)$; PAD: pressão arterial diastólica ( $\mathrm{mmHg})$; PAS: pressão arterial sistólica $(\mathrm{mmHg})$;

a. diferença significativa para o grupo 20-29; b. diferença significativa para o grupo 30-39; c. diferença significativa para o grupo 40-49. F: ANOVA one way, com post hoc de Bonferroni; $p<0,05$

Tabela 2. Caracterização amostral das mulheres, com valores de média e desvio padrão, conforme as categorias de idade.

\begin{tabular}{ccccccc}
\hline & \multicolumn{7}{c}{ Idade } \\
\cline { 2 - 7 } & $\begin{array}{c}\mathbf{2 0 - 2 9} \\
\mathbf{( N = 8 6 3 )}\end{array}$ & $\begin{array}{c}\mathbf{3 0 - 3 9} \\
\mathbf{( N = 8 8 1 )}\end{array}$ & $\begin{array}{c}\mathbf{4 0 - 4 9} \\
\mathbf{( N = 9 7 8 )}\end{array}$ & $\begin{array}{c}\mathbf{5 0 - 5 9} \\
\mathbf{( N = 6 9 3 )}\end{array}$ & $\mathbf{F}$ & $\mathbf{p}$ \\
\hline Estatura & $163,5 \pm 7,2$ & $162,14 \pm 7,5^{\mathrm{a}}$ & $161,0 \pm 7,2^{\mathrm{a}, \mathrm{b}}$ & $160,0 \pm 7,5^{\mathrm{a}, \mathrm{b}, \mathrm{c}}$ & 34,05 & 0,000 \\
MC & $61,0 \pm 10,8$ & $65,59 \pm 12,6^{\mathrm{a}}$ & $68,0 \pm 12,7^{\mathrm{a}, \mathrm{b}}$ & $69,8 \pm 12,5^{\mathrm{a}, \mathrm{b}, \mathrm{c}}$ & 79,44 & 0,000 \\
IMC & $22,7 \pm 3,4$ & $24,8 \pm 4,1^{\mathrm{a}}$ & $26,2 \pm 4,5^{\mathrm{a}, \mathrm{b}}$ & $27,2 \pm 4,3^{\mathrm{a}, \mathrm{b}, \mathrm{c}}$ & 177,42 & 0,000 \\
PAD & $70,27 \pm 10,1$ & $72,46 \pm 10,6^{\mathrm{a}}$ & $75,5 \pm 11,6^{\mathrm{a}, \mathrm{b}}$ & $79,5 \pm 12,1^{\mathrm{a}, \mathrm{b}, \mathrm{c}}$ & 84,13 & 0,000 \\
PAS & $111,14 \pm 13,7$ & $112,51 \pm 13,6$ & $116,6 \pm 15,4^{\mathrm{a}, \mathrm{b}}$ & $123,5 \pm 18,1^{\mathrm{a}, \mathrm{b}, \mathrm{c}}$ & 85,95 & 0,000
\end{tabular}

N: número de indivíduos; Estatura (cm) MC: Massa Corporal (kg); IMC: índice de massa corporal (kg/m²); PAD: pressão arterial diastólica ( $\mathrm{mmHg}$ ); PAS: pressão arterial sistólica ( $\mathrm{mmHg}$ );

a. diferença significativa para o grupo 20-29; b. diferença significativa para o grupo 30-39; c. diferença significativa para o grupo 40-49. F: ANOVA one way, com post hoc de Bonferroni; $p<0,05$ 
A prevalência de indivíduos com hipertensão foi de $19,4 \%$ nos homens e $15,9 \%$ nas mulheres, e cabe ressaltar que $9,5 \%$ e $6,6 \%$ entre homens e mulheres, respectivamente, foram classificados com pressão arterial limítrofe. Na tabela 3 destaca-se, por meio da proporção, a apresentação de indivíduos considerados com pressão arterial normal, limítrofe e elevada, segundo a classificação do estado nutricional propostos pelos pontos de corte do IMC e faixas etárias para ambos os sexos.

Tabela 3. Distribuição (\%) dos pontos de corte do IMC de acordo com faixas etárias em ambos os sexos.

\begin{tabular}{l|ccc|ccc}
\hline \multirow{3}{*}{ Idade } & \multicolumn{3}{c}{ Homens } \\
\cline { 2 - 7 } & Eutrófico \% & Sobrepeso \% & Obeso \% & Eutrófico \% & Sobrepeso \% & Obeso \% \\
\hline $\mathbf{2 0 - 2 9}$ & 64,5 & 27,5 & 8,0 & 78,0 & 17,8 & 4,2 \\
$\mathbf{3 0 - 3 9}$ & 43,6 & 44,2 & 12,2 & 58,0 & 30,5 & 11,5 \\
$\mathbf{4 0 - 4 9}$ & 30,1 & 48,3 & 21,6 & 44,3 & 37,6 & 18,1 \\
$\mathbf{5 0 - 5 9}$ & 26,9 & 49,6 & 23,5 & 32,6 & 46,3 & 24,1 \\
\hline
\end{tabular}

\%: porcentagens dos indivíduos;

Pontos de Corte IMC: eutrófico: entre 18,5 kg/ $/ \mathrm{m}^{2}$ a $24,99 \mathrm{~kg} / \mathrm{m}^{2}$; sobrepeso: entre $25 \mathrm{~kg} / \mathrm{m}^{2}$ a $29,99 \mathrm{~kg} / \mathrm{m}^{2}$; obesidade: acima de $30 \mathrm{~kg} / \mathrm{m}^{2}$

$\mathrm{Na}$ tabela 4 se refere às razões de chance para - de hipertensão arterial segundo os pontos de referência: sexo feminino, faixa etária entre 20 a 29 anos e os sujeitos eutróficos.

Tabela 4. Razão de chances de hipertensão arterial diastólica e sistólica conforme sexo, idade e IMC

\begin{tabular}{ccccc}
\hline Variáveis & $\begin{array}{c}\text { Hipertensão Diastólica } \\
\text { (IC95\%) }\end{array}$ & $\mathbf{p}$ & $\begin{array}{c}\text { Hipertensão Sistólica } \\
\text { (IC95\%) }\end{array}$ & $\mathbf{p}$ \\
\hline $\begin{array}{c}\text { Sexo } \\
\text { Feminino }\end{array}$ & 1 & & 1 & \\
Masculino & $1,97(1,61-2,41)$ & 0,000 & $1,97(1,57-2,47)$ & 0,000 \\
Idade & 1 & & 1 & \\
$20-29,99$ & $1,28(0,87-1,90)$ & 0,204 & $1,05(0,66-1,67)$ & 0,813 \\
$30-39,99$ & $1,93(1,33-2,78)$ & 0,000 & $1,42(0,92-2,20)$ & 0,105 \\
$40-49,99$ & $2,89(1,98-4,22)$ & 0,000 & $3,15(2,05-4,82)$ & 0,000 \\
$50-59,99$ & & & & \\
IMC & 1 & & 1 & 0,000 \\
Normal & $2,09(1,63-2,68)$ & 0,000 & $2,12(1,60-2,80)$ & 0,000 \\
Sobrepeso & $4,60(3,51-6,03)$ & 0,000 & $4,15(3,05-5,64)$ & \\
Obeso & & & \\
\hline IC= Intervalo de confianç; IMC= índice de massa corporal; ${ }^{*} p<0,05$. &
\end{tabular}

$I C=$ Intervalo de confiança; $I M C=$ índice de massa corporal; * $p<0,05$.
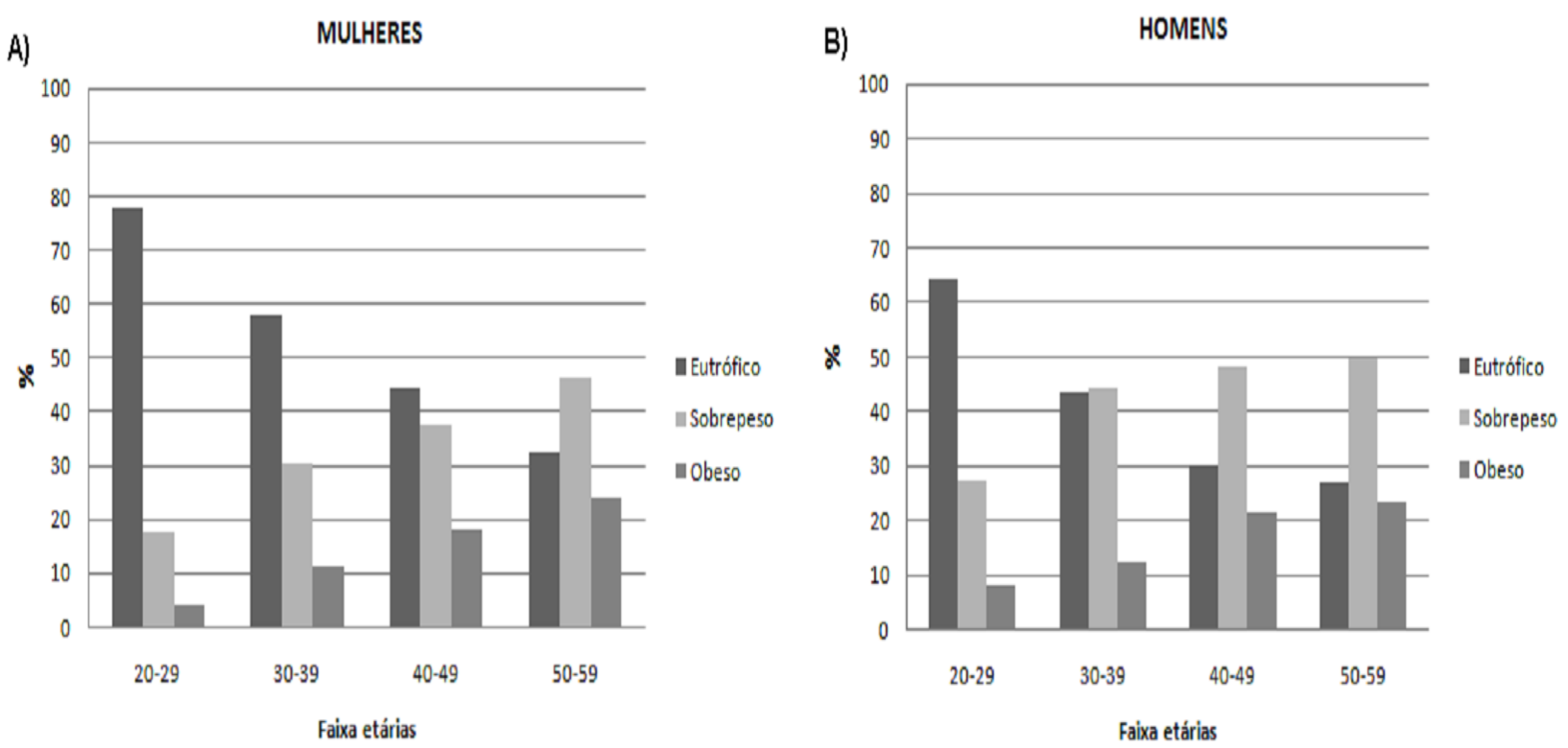

A) Distribuição do estado nutricional para as Mulheres; B) Distribuição do estado nutricional para os Homens; \%: porcentagens dos indivíduos pelo estado nutricional; Pontos de Corte IMC: eutrófico: entre 18,5 kg/ $/ \mathrm{m}^{2}$ a 24,99 kg/ $\mathrm{m}^{2}$; sobrepeso: entre $25 \mathrm{~kg} / \mathrm{m}^{2}$ a $29,99 \mathrm{~kg} / \mathrm{m}^{2}$; obesidade: acima de $30 \mathrm{~kg} / \mathrm{m}^{2}$

Figura 1. Distribuição (\%) dos pontos de corte do IMC de acordo com faixas etárias em ambos os sexos. 


\section{Discussão}

Os resultados do presente estudo indicam que a partir dos quarenta anos de idade verifica-se aumento na prevalência de indivíduos com pressão arterial elevada para ambos os sexos, principalmente entre os considerados com sobrepeso e obesidade.

Ao observar o estado nutricional para ambos os sexos, a porcentagem de indivíduos eutróficos apresentou diminuição a cada década de vida. Ao contrário do sobrepeso que atinge valores alarmantes, acima de $44 \%$ para os homens e para as mulheres acima dos $30 \%$. Em estudo realizado por Flegal et al. (2002), também observaram aumento exponencial de indivíduos acima do peso a partir da década de oitenta até o presente momento, corroborando com o presente estudo de corte transversal que também verificou aumentos substanciais de indivíduos acima do peso.

Quanto à prevalência de obesidade com o passar dos anos, os valores já chegam a $20 \%$ para ambos os sexos por volta dos quarenta anos. Tal aumento de indivíduos obesos pode ser verificada no estudo de Flegal et al. (2002) que por meio de dados do NHANES verficaram uma tendência crescente de indivíduos obesos entre 20 e 74 anos, sendo que para os homens este aumento foi de $27,7 \%$ e para as mulheres de $34 \%$ desde o início das suas coletas.

A Sociedade brasileira de cardiologia (2006) alega que o aumento do peso corporal pode ser considerado um fator predisponente para 0 desenvolvimento da hipertensão arterial, sendo responsável por $20 \%$ a $30 \%$ dos casos da pressão arterial elevada; em que $75 \%$ dos homens e $65 \%$ das mulheres apresentam hipertensão diretamente atribuível ao excesso de peso. Vasan et al. (2001) corrobora com esta afirmação por meio do estudo de Framingham, ressaltando que a hipertensão arterial aumenta em até duas vezes em homens e três vezes nas mulheres 0 desenvolvimento de problemas cardiovasculares, principalmente entre os que estão acima do peso. Isto foi diferente dos nossos resultados que apontam para uma razão de chances maior para os homens de quase duas vezes mais chances do que as mulheres de apresentar hipertensão arterial tanto diastólica quanto sistólica.
Esta preocupação pode ser observada na pesquisa de Ogden et al. (2006) com a população americana de adultos, que apresentam ascensão de $28 \%$ de hipertensos entre os obesos a partir dos 20 aos 39 anos e $31 \%$ entre os de 40 a 59 anos. Apesar da hipertensão não apresentar manifestações quando em estágios iniciais (2006), os portadores desta, muitas vezes desconhecem as consequências que norteiam este problema de saúde, mesmo tendo recebido orientação de algum profissional da saúde (PÉRESA; MAGNAA; VIANAB, 2003).

Com isto, a prevalência de indivíduos hipertensos verificado na população brasileira por meio de estudos descritivos publicados nas diretrizes da Sociedade brasileira de cardiologia (2006) aponta que $22,3 \%$ a $43,9 \%$ dos adultos brasileiros apresentam pressão arterial elevada. Juntamente com estes dados, a Sociedade brasileira de cardiologia (2006) destaca relatos da World health organization (2000) no qual os pontos de corte do IMC $\left(25,0\right.$ e $\left.30,0 \mathrm{~kg} / \mathrm{m}^{2}\right)$ estão fortemente associados com risco de morbidade por meio das doenças cardiovasculares associadas com a idade. Estes estudos vão de encontro com os resultados da presente pesquisa, em que se verifica 0 aumento da prevalência de indivíduos hipertensos, principalmente entre os obesos para as mulheres em todas as idades e a partir dos 40 anos para os homens (tabela 4).

Fato este também verificado nos estudos do NHANES no qual foi relatado que $29 \%$ dos adultos americanos são hipertensos, sendo que $69 \%$ não controlam a doença (SCHOCKEN et al., 2008). Assim como, Deshmukh et al. (2006) revelaram a relação positiva entre as porcentagens de $21,8 \%$ para homens e $19,8 \%$ para as mulheres asiáticas que estão acima do peso com a hipertensão. A literatura ainda cita que a pressão arterial aumenta com a idade, principalmente a partir do sexto decênio de vida (CHOBANIAN, 2003), o que não foi verificado em nosso estudo.

Com relação aos pontos de corte do IMC para o sobrepeso e obesidade, a razão de chances encontrada nos nossos resultados é similar aos achados de Greenlund, Croft e Mensah (2004) que apresentam uma razão de chances do desenvolvimento de hipertensão arterial de OR: 3,34 e OR: 4,42, respectivamente para ambos os sexos. Já quando destacado o termo pré- 
hipertensão, o mesmo estudo descreve uma associação de OR: 1,46 em indivíduos com sobrepeso e OR: 2,26 para os obesos. Outro estudo realizado por Wilsgaard, Schirmer e Arnesen (2000) demonstra que as mudanças da pressão arterial podem chegar a uma vez e meia para ambos os sexos quando associadas com o estado nutricional.

Por conseguinte, algumas limitações são levadas em consideração no presente estudo como o caráter particular da amostra de indivíduos, ou seja, cidadãos do município de Curitiba, estado do Paraná que por sua vez limita a extrapolação dos dados observados; o delineamento transversal do estudo não garante a precedência temporal das variáveis sobre a prevalência da hipertensão arterial; outro fator se refere ao diagnóstico de hipertensão arterial, medida esta baseada em uma única mensuração, uma vez que o ideal seria realizar a média de duas medidas de pressão arterial obtidas em dois momentos distintos ( $\underline{\mathrm{CHOBANIAN}}, 2003$ ).

Outras variáveis poderiam ser avaliadas e controladas para melhor inferência dos resultados como: controle de medicamentos, momentos de estresse, o controle do nível de atividade física, diversidade demográfica, o consumo de alguns alimentos ou bebidas que podem interferir na variação da PA entre outros.

Contudo, os resultados denotam a importância da incorporação de políticas públicas direcionadas ao controle do sobrepeso/obesidade, que por sua vez acarreta o aparecimento da hipertensão arterial. Assim como, estes achados apontam para novos estudos que podem ser realizados para avaliar especificamente as modificações hemodinâmicas em indivíduos acima do peso, abrindo caminho para estratégias terapêuticas mais específicas para este grupo de indivíduos.

\section{Referências}

CHIRINOS, J. A. et al. Body mass index and hypertension hemodynamic subtypes in the adult US population. Archives of Internal Medicine, Chicago, v. 169, n. 6, p. 580-586, 2009.

CHOBANIAN, A. V. (Org.). The seventh report of the joint national committee on prevention, detection, evaluation, and treatment of high blood pressure: the JNC 7 report. Journal American
Medicine Association, Chicago, v. 289, n. 19, p. 2560-2572, 2003.

DESHMUKH, P. R. et al. Relationship of anthropometric indicators with blood pressure levels in rural Wardha. Indian Journal of Medical Research, New Delhi, v. 123, p. 657-664, 2006.

FERREIRA, I. et al. Amsterdam growth and health longitudinal study: the metabolic syndrome, cardiopulmonary fitness, and subcutaneous trunk fat as independent determinants of arterial stiffness. Archives of Internal Medicine, Chicago, v. 165, n. 8, p. 875-882, 2005.

FLEGAL, K. M. et al. Prevalence and trends in obesity among US adults, 1999-2000. Journal American Medicine Association, Chicago, v. 288, p. 1723-1727, 2002.

GREENLUND, K. J.; CROFT, J. B.; MENSAH, G. A. Prevalence of heart disease and stroke risk factors in persons with prehypertension in the United States, 1999-2000. Archives of Internal Medicine, Chicago, v. 164, p. 2113-2118, 2004.

LOHMAN, T. G.; ROCHE, A. F.; MARTORELL, R. Anthropometric standardization reference manual. Champaign: Human Kinetics; 1988.

OGDEN, C. L. et atl. Prevalence of overweight and obesity in the United States, 1999-2004. Journal American Medicine Association, Chicago, v. 295, p. 1549-1555, 2006.

PÉRESA, D. S.; MAGNAA, J. M.; VIANAB, L. A. Portador de hipertensão arterial: atitudes, crenças, percepções, pensamentos e práticas. Revista de Saúde Pública, São Paulo, v. 37, n. 5, p. 635-642, 2003.

POLANCZIK, C. A. Decisão clínica em hipertensão arterial sistêmica baseada em análises econômicas. Revista Brasileira de Hipertensão, Ribeirão Preto, v. 9, n. 1, p. 29-34, 2002.

SAFAR, M. E.; CZERNICHOW, S.; BLACHER, J. Obesity, arterial stiffness, and cardiovascular risk. Journal of the American Society of Nephrology, Washington, v. 17, n. 4, p. S109S111, 2006.

STELFOX, H. T. et al. Hemodynamic monitoring in obese patients: the impact of body mass index on cardiac output and stroke volume. Critical Care Medicine, Mount Prospect, v. 34, n. 4, p. 1243-1246, 2006. 
SCHILLACI, G. et al. Metabolic syndrome is associated with aortic stiffness in untreated essential hypertension. Hypertension,

Hagerstown, v. 45, n. 6, p. 1078- 1082, 2005.

SCHOCKEN, D. D. et al. Prevention of heart failure: a scientific statement from the american heart association councils on epidemiology and prevention, clinical cardiology cardiovascular nursing, and high blood pressure research; quality of care and outcomes research interdisciplinary working group; and functional genomics and translational biology interdisciplinary working group. Circulation, Boston, v. 117, p. 2544-2565, 2008. Disponível em:

<http://www.ncbi.nlm.nih.gov/pubmed/18391114>. Acesso em: 17 abr. 2011.

SCUTERI, A. et al. Metabolic syndrome amplifies the ageassociated increases in vascular thickness and stiffness. Journal of the American College of Cardiology, Washington, v. 43, n. 8, p.13881395, 2004.

\section{SOCIEDADE BRASILEIRA DE CARDIOLOGIA} (SBC). Diretrizes brasileiras de hipertensão arterial, V. São Paulo: SBH, 2006. Disponível em: $<$ http://bvsms.saude.gov.br/bvs/publicacoes/v dir etrizes brasileira hipertensao arterial 2006.pdf>. Acesso em: 17 abr. 2011.

VASAN, R. S. et al. Assessment of frequency of progression to hypertension in non-hypertensive participants in the Framingham Heart Study: a cohort study. The Lancet, Atlanta, v. 358, p. 1682-1686, 2001.

WILSGAARD, T.; SCHIRMER, H.; ARNESEN, E. Impact of body weight on blood pressure with a focus on sex differences: the tromso study, 19861995. Archives of Internal Medicine, Chicago, v. 160, p. 2847-2853, 2000.

WORLD HEALTH ORGANIZATION (WHO). The asia-pacific perspective: redefining obesity and its treatment. Geneva, Switzerland: World Health Organization, 2000.

\section{Endereço:}

Anderson Zampier Ulbrich.

Núcleo de Cardiologia do CEFID/UDESC

Rua Pascoal Simone, 358 Coqueiros

Florianópolis SC Brasil

88080-350

Telefone: (48) 3321-8643

e-mail: anderson_u@hotmail.com
Recebido em: 2 de junho de 2010. Aceito em: 20 de abril de 2011.

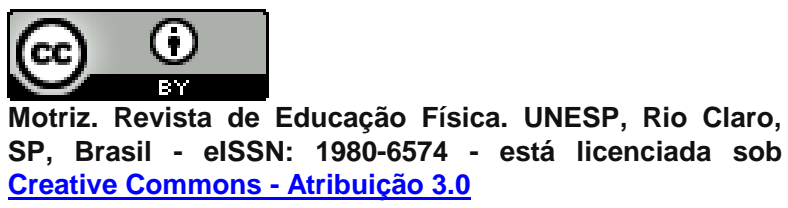
Creative Commons - Atribuição 3.0 